\title{
A UNIFIED APPROACH TO INTERFACE AND CONFINED BULK OPTICAL PHONONS IN QUANTUM WELLS AND SUPERLATTICES
}

\author{
J. RöSELER AND D. SUISKY \\ Institute of Theoretical Physics, Humboldt-University Berlin \\ Invalidenstr. 110, D-O-1040 Berlin, Federal Republic of Germany
}

A unified description of confined optical LO, TO and interface phonons is given on the basis of vector "ghost" fields defined in the whole space. These fields are characterized by the relations for longitudinal and transversal fields. Real displacement fields are obtained by introducing confinement conditions. Interface charges and vortices appear due to this procedure.

PACS numbers: 73.20.Dx

\section{Introduction}

Optical phonon modes in quantum wells (QW) and superlattices (SL) have been calculated by several authors [1-13] on the basis of classical theory of phonon modes for bulk materials and the electrodynamics. The equation of motion is

$$
\ddot{u}=-\omega_{\text {To }}^{2} u+(e / M) \boldsymbol{E},
$$

where $\boldsymbol{u}, \boldsymbol{E}$, and $M$ are the mechanical displacement field, the long-range electric field and the reduced mass of the unit cell, respectively. Equation (1) has to be solved using the boundary conditions for $\boldsymbol{E}$ and the dielectric displacement field $\boldsymbol{D}$ at the interfaces of the QW and the SL. Mechanical and electric fields are related additionally by

$$
\boldsymbol{P}=N e \boldsymbol{u} \text {. }
$$

The polarization field and the density of unit cells are denoted by $\boldsymbol{P}$ and $N$, respectively. Neglecting retardation effects one obtains

$$
\operatorname{rot} \boldsymbol{E}=0 \text {, }
$$

i.e., the electric field is purely longitudinal. It follows from equation of motion (1) that $u$ may have a transversal component.

Equation (1) has been generalized by Barbiker [14] by including additional terms which describe long-range mechanical forces being connected with the $x$-dependence of $\boldsymbol{u}$ :

$$
\ddot{u}+\omega_{\text {TO }}^{2} u+c_{l}^{2} \operatorname{grad} \operatorname{div} u-c_{\mathrm{t}}^{2} \operatorname{rot} \operatorname{rot} u-(e / M) \boldsymbol{E}=0 .
$$


Equation (1) can be derived immediately from (4) by assuming

$$
\begin{aligned}
\operatorname{div} u & =0, \\
\operatorname{rot} u & =0 .
\end{aligned}
$$

The difference between (1) and (4) is striking. From an equation of type (1) interface modes (IF) can be derived, whereas Eq. (4) gives confined bulk modes (CB) as solutions after introducing confinement conditions. Relations (5) and (6) may be considered as criteria for the structure of IF mechanical displacement fields. Moreover, (5) and (6) may be the starting point for the calculation of the mechanical displacement fields without invoking electrodynamical boundary conditions. Then, it seems to be quite naturally to define the displacement fields of CB modes by relations of the same type:

$$
\begin{array}{ll}
\operatorname{div} u \neq 0, & \operatorname{rot} u=0, \\
\operatorname{div} u=0, & \operatorname{rot} u \neq 0 .
\end{array}
$$

Using (7) and (8) the equation of motion (4) is specialized for longitudinal and transversal CB modes, respectively.

Definitions (5) to (8) are independent of the geometrical form of the interface separating different materials. They can be assumed to be valid for QW, SL or quantum wires. Furthermore, it can be shown that the $u$ fields derived from relations (5) to (8) give together with the equation of motion such fields $\boldsymbol{E}$ and $\boldsymbol{D}$ which are consistent with the electrodynamical boundary conditions.

\section{Interface modes}

\subsection{Planar interfaces ( $Q W$ and $S L)$}

For planar interfaces the operators grad, div and rot are used in cartesian coordinate systems. Assuming a single interface between two different materials the relations (5) and (6) are fulfilled by the vector fields

$$
\begin{aligned}
& u_{1}=a(q)(-\mathrm{i} q / q, 1) \exp (-q z) \exp (\mathrm{i} q x), \\
& u_{2}=b(q)(-\mathrm{i} q / q,-1) \exp (q z) \exp (\mathrm{i} q x),
\end{aligned}
$$

where $q$ is the wave vector parallel to the interface which is assumed to be localized at $z=0$. Expressions (9) and (10) are divergent for $z \rightarrow \infty$ and $z \rightarrow-\infty$, respectively. Therefore, we cannot identify (9) and (10) with the real displacement fields. The real fields can be defined by introducing confinement conditions which restrict the existence of the fields to that halfspace where they remain finite. Consequently, in contrast to the real fields (9) and (10) are qualified as "ghost" fields and will be denoted by $\boldsymbol{u}^{\prime}$ in the following. If the left halfspace is empty, the polarization field is given by

$$
\boldsymbol{P}=\Theta(z) \boldsymbol{P}^{\prime} .
$$

According to (2) the "ghost" field $\boldsymbol{P}^{\prime}$ is defined by

$$
\boldsymbol{P}^{\prime}=N e \boldsymbol{u}^{\prime}
$$


fulfilling the conditions

$$
\operatorname{div} \boldsymbol{P}^{\prime}=0, \quad \operatorname{rot} \boldsymbol{P}^{\prime}=0 .
$$

Using (11) and the relations

$$
\operatorname{rot} \boldsymbol{E}=0, \quad \operatorname{div} D=0, \quad D=E+4 \pi P,
$$

the fields $\boldsymbol{E}, \boldsymbol{D}$ and $\boldsymbol{P}$ of an interface mode travelling in the right halfspace can be calculated. One obtains

$$
\begin{aligned}
& \boldsymbol{E}=2 \pi \sigma(q)(\mathrm{i} q / q,-\operatorname{sgn}(z)) \exp (-q|z|) \exp (\mathrm{i} q x), \\
& \boldsymbol{P}=\Theta(z) \sigma(q)(-\mathrm{i} q / q, 1) \exp (-q z) \exp (\mathrm{i} q x), \\
& D=2 \pi \sigma(q)(-\mathrm{i} q \operatorname{sgn}(z) / q, 1) \exp (-q|z|) \exp (\mathrm{i} q x) .
\end{aligned}
$$

Polarization charges exist at the interface at $z=0$ and do not appear in the bulk. This follows from

$$
\begin{aligned}
& \operatorname{div} \Theta(z) \boldsymbol{P}^{\prime}=\boldsymbol{P}^{\prime} \operatorname{grad} \Theta(z)=\boldsymbol{P}^{\prime}(0,0, \delta(z)), \\
& \operatorname{rot} \Theta(z) \boldsymbol{P}^{\prime}=\boldsymbol{P}^{\prime} \times \operatorname{grad} \Theta(z)=\boldsymbol{P}^{\prime} \times(0,0, \delta(z)) .
\end{aligned}
$$

Sources and vortices appear together, but only at the interface. The IF modes are neither longitudinal nor transversal. The boundary conditions of the electrodynamic are fulfilled by (15) to (17).

\subsection{Quantum wires}

Recently, the procedure discussed in Sec. 2.1 has been used in order to derive IF modes of a quantum wire [15]. Assuming (5) and (6), it is readily seen that one can make the ansatz

$$
u=\operatorname{grad} \Phi,
$$

obtaining with the help of (5)

$$
\Delta \Phi=0,
$$

where the Laplace operator is given in cylindrical coordinates. The solutions of (21) are the modified Bessel functions $I_{m}(k r)$ and $K_{m}(r k)$, giving the wire "ghost" fields.

\section{Dispersion relations}

The dispersion relations of IF modes of a halfspace, the double heterostructure (DHS) and the SL can be expressed in the unified form

$$
\left[\varepsilon_{1}(\omega)+\varepsilon_{2}(\omega)\right] /\left[\varepsilon_{1}(\omega)-\varepsilon_{2}(\omega)\right]=\eta(q, k) .
$$

The function $\eta(q, k)$ depends on the components of the wave vector $Q=(q, k)$ parallel and perpendicular to the interface. It holds: $\eta=0$ for the halfspace, $\eta= \pm \exp (-q d)$ for the DHS and

$$
\eta(q, k)=\left[\cos h q\left(d_{1}-d_{2}\right)-\cos k d\right] /\left[\cos h q\left(d_{1}+d_{2}\right)-\cos k d\right]
$$

for the SL. 


\section{Confined bulk modes}

\subsection{Symmetric modes}

Repeating the same procedure as in Sec. 2.1 concerning relations (7) and (8) we obtain the CB mode "ghost" fields

$$
\begin{aligned}
& u_{3}=a(q)\left(\mathrm{i} e_{q} f(z)+e_{z} g(z)\right) \exp (\mathrm{i} q x), \\
& u_{4}=b(q)\left(\mathrm{i} e_{q} g(z)+e_{z} f(z)\right) \exp (\mathrm{i} q x) .
\end{aligned}
$$

$\boldsymbol{e}_{q}$ and $\boldsymbol{e}_{z}$ are the unit vectors parallel and perpendicular to the interface, respectively. Assuming (24) as a longitudinal and (25) as a transversal mode we obtain from relation $\operatorname{div} u_{4}^{\prime}=0$ :

$$
-q g(z)+f^{\prime}(z)=0 .
$$

Relation (26) can be fulfilled with the ansatz

$$
g(z)=\alpha \cos \lambda z, \quad f(z)=\beta \sin \lambda z,
$$

which gives

$$
\begin{aligned}
& u_{3}^{\prime}=A(q)\left(\mathrm{i} e_{q} \sin \lambda z+e_{z}(\lambda / q) \cos \lambda z\right) \exp (\mathrm{i} q x), \\
& u_{4}^{\prime}=B(q)\left(\mathrm{i}_{q}(\lambda / q) \cos \lambda z+e_{z} \sin \lambda z\right) \exp (\mathrm{i} q x) .
\end{aligned}
$$

Real polarization fields are now introduced by applying the confinement conditions

$$
\boldsymbol{P}_{\mathbf{3}}=\boldsymbol{P}_{\mathbf{3}}^{\prime} \Lambda(z), \quad \boldsymbol{P}_{\mathbf{4}}=\boldsymbol{P}_{4}^{\prime} \Lambda(z)
$$

with $\Lambda(z)=\Theta(z+a / 2)-\Theta(z-a / 2), a$ being the layer thickness. The values of (30) are not completely determined by (28) and (29). The symmetry of the DHS or the SL allows two different choices of the $z$-component of $\boldsymbol{u}^{\prime}$ at the interfaces:

case (a) $g(+a / 2)=g(-a / 2)=0, \quad f(+a / 2)=-f(-a / 2) \neq 0$

or

$$
\text { case (b) } g(+a / 2)=g(-a / 2) \neq 0, \quad f(+a / 2)=f(-a / 2)=0 .
$$

From (31) and (32) we obtain a relation for $\lambda$ :

$$
\begin{aligned}
& \text { case (a) } \quad \lambda=\pi K / a, \quad K=1,3,5 \ldots \\
& \text { case (b) } \quad \lambda=\pi K / a, \quad K=2,4,6 \ldots
\end{aligned}
$$

\subsection{Antisymmetric modes}

Repeating the procedure of the Sec. 4.1 the sin- and cos-functions change their places in the expression for the displacement field and another prefactors appear. 


\section{Nonuniqueness of the potentials}

Relations (7) and (8) do not determine the real fields unambiguously because we can add a gradient to $\Lambda(z) u^{\prime}$ and $\operatorname{rot} u=0$ remains valid, i.e.

$$
u=\Lambda(z) u^{\prime}+\operatorname{grad} \Phi
$$

and $\operatorname{div} u=0$ holds also for a field

$$
u=\Lambda(z) u^{\prime}+\operatorname{rot} a \text {. }
$$

It follows from (7) and (35)

$$
\operatorname{div} u=\Lambda(z) \operatorname{div} u^{\prime}+u_{z}^{\prime}(\delta(z+\dot{a} / 2)-\delta(z-a / 2))+\Delta \Phi .
$$

Choosing the potential so that

$$
\Delta \Phi=-u_{z}^{\prime}(\delta(z+a / 2)-\delta(z-a / 2))
$$

is valid, a compensation of the interface sources in (37) would appear. This compensation plays a role in case (b) if the longitudinal CB mode has a source-like IF contribution. In the case (a) the modes would not have a source-like but a vortex-like IF contribution which is in disagreement with the classification of the modes and cannot be compensated by means of potentials.

By choosing $\Delta \Phi=0$ there is no compensation in (37). Now, the longitudinal CB mode has a source-like IF contribution in the case (b) and a vortex-like IF contribution in the case (a). The discontinuity of one of the components of the fields (28) and (29) (either the $x$-, $y$ - or the $z$-component) cannot be removed at all, if one describes the fields by functions of $\cos \lambda z$ or $\sin \lambda z$ type.

\section{References}

[1] M.V. Klein, IEEE J. Quantum Electron. 22, 1760 (1986).

[2] B. Jusserand, M. Cardona, in: Light Scattering in Solids V, Ed. M. Cardona, G. Guentherodt, Springer-Verlag, Berlin 1989.

[3] M. Cardona, Superlattices Microstruct. 5, 27 (1989).

[4] J. Menendez, J. Lumin. 44, 285 (1989).

[5] S. M. Rytov, Zh. Eksp. Teor. Fiz. 29, 605 (1955).

[6] E.P. Pokatilov, S.I. Beril, Phys. Status Solidi B 118, 567 (1983).

[7] R.E. Camley, D.L. Mills, Phys. Rev. B 29, 1695 (1984).

[8] R. Lassnig, Phys. Rev. B 30, 7132 (1984).

[9] V.M. Fomin, E.P. Pokatilov, Phys. Status Solidi B 132, 69 (1985).

[10] L. Wendler, Phys. Status Solidi B 129, 513 (1985).

[11] F. Bechstedt, R. Enderlein, Phys. Status Solidi B 131, 53 (1985).

[12] F. Bechstedt, G. Gerecke, Phys. Status Solidi B 154, 565 (1989).

[13] R. Enderlein, D. Suisky, J. Roeseler, Phys. Status Solidi B 165, 9 (1991).

[14] M. Barbiker, J. Phys. C 19, 683 (1986); Physica B/C 145, 111 (1987).

[15] R. Enderlein, to be published. 